\title{
Efficient Structural Joins on Indexed XML Documents
}

\author{
Shu-Yao Chien \\ NCR/Teradata Division \\ sc180001@ncr.com
}

\author{
Zografoula Vagena \\ CSE Dept., UC Riverside \\ foula@cs.ucr.edu
}

\author{
Donghui Zhang \\ CSE Dept., UC Riverside \\ donghui@cs.ucr.edu
}

\author{
Vassilis J. Tsotras* \\ CSE Dept., UC Riverside \\ tsotras@cs.ucr.edu
}

Carlo Zaniolo*

CS Dept. UCLA

zaniolo@cs.ucla.edu

\begin{abstract}
Queries on XML documents typically combine selections on element contents, and, via path expressions, the structural relationships between tagged elements. Structural joins are used to find all pairs of elements satisfying the primitive structural relationships specified in the query, namely, parent-child and ancestordescendant relationships. Efficient support for structural joins is thus the key to efficient implementations of XML queries. Recently proposed node numbering schemes enable the capturing of the XML document structure using traditional indices (such as $\mathrm{B}+$-trees or R-trees). This paper proposes efficient structural join algorithms in the presence of tag indices. We first concentrate on using $\mathrm{B}+-$ trees and show how to expedite a structural join by avoiding collections of elements that do not participate in the join. We then introduce an enhancement (based on sibling pointers) that further improves performance. Such sibling pointers are easily implemented and dynamically maintainable. We also present a structural join algorithm that utilizes R-trees. An extensive experimental comparison shows that the $\mathrm{B}+$-tree structural joins are more robust. Furthermore, they provide drastic improvement gains over the current state of the art.
\end{abstract}

This research was supported by NSF grants IIS-9907477, EIA-9983445, IIS-0070135, and the Dept. of Defense.

Permission to copy without fee all or part of this material is granted provided that the copies are not made or distributed for direct commercial advantage, the VLDB copyright notice and the title of the publication and its date appear, and notice is given that copying is by permission of the Very Large Data Base Endowment. To copy otherwise, or to republish, requires a fee and/or special permission from the Endowment.

Proceedings of the 28th VLDB Conference, Hong Kong, China, 2002

\section{Introduction}

The problem of managing and querying XML documents efficiently poses interesting challenges for database researchers. XML documents can have a rather complex internal structure; in fact, an XML document can be viewed as an ordered tree. Tree nodes correspond to document elements (or attributes) while edges represent direct element-subelement relationships. This tree-centric representation is apparent in XML languages like Quilt [8], XQuery [39] and XPath [38], which qualify documents for retrieval both by their structure and the values in their elements. For instance, a value-based selection for a document can be specified by conditions on its elements' names (tags), their attributes, and the text strings (i.e., PCDATA) contained in such elements. A structure-based selection instead relies on the structural relationships, namely: parent-child and ancestor-descendant relationships. For example, the query:

section[title="Overview"]//figure[caption="R-tree"]

finds all figures with caption 'R-tree' under sections whose title is "Overview". This complex query consists of three conditions:

- the conditions section[title="Overview"] and figure[caption="R-tree"] are value-based since they select document elements using their values or contents, while

- the double slash in section//figure corresponds to a structural condition, in particular, an ancestor-descendant relationship. It is a shorthand for a path expression specifying that there must be a path leading from the first element to the second one (i.e., the second element must be a descendant of the first in the document tree).

Using path expressions, users are allowed to navigate through arbitrary long paths in the tree. A single slash in the query (section/figure), would find only those figures that are children of section elements (i.e., a parent-child relationship). 
Traditional indexing schemes, such as B+-trees, can be easily extended to support value based queries on XML documents. Path expression queries pose a much harder problem, requiring the computation of structural joins $[1,24]$. Previously proposed structural join algorithms assume that the ancestor and the descendant elements are provided before the join in two ordered lists [1, 24]. However, that implies that all ancestor and descendant elements are accessed either from indices or even from physical data pages before the join. That may cause unnecessary I/O and slow down the join process. Instead we assume that an index exists in each of the joined lists. Typically, list elements belong to the same document tag (sections, figures, chapters, etc.) and tag indices are easily maintained [11]. Moreover, such indices can resolve element relationships by utilizing recently proposed numbering schemes that capture the document structure $[24,1,11]$.

This paper proposes efficient structural join algorithms in the presence of tag indices. First we concentrate on using $\mathrm{B}+$-trees (with numbering schemes) and show how to take advantage of the available structural information before joining, to filter out and minimize unnecessary data reads. We then introduce a simple enhancement that further improves performance by adding sibling pointers based on the notion of "containment". Such pointers are easily implemented and can be maintained dynamically. We also present a structural join algorithm that utilizes R-trees. An extensive experimental comparison shows that the $\mathrm{B}+-$ tree structural join enhanced with few sibling pointers provides the most robust solution. Furthermore, this approach can provide drastic improvement gains over the current state of the art.

The main contributions of this paper are summarized below:

- An efficient B+-tree based structural join algorithm is presented;

- The utilization of sibling pointers is introduced for further performance gains;

- An extensive experimental section is provided, comparing the above algorithms with R-tree based structural joins as well as non-index based joins.

The rest of the paper is organized as follows. Section 2 reviews related work. Section 3 proposes the $\mathrm{B}+$-tree based structural join algorithms, while section 4 discusses the R-tree based structural join. Problem variations are examined in Section 5 while Section 6 presents performance results and Section 7 concludes the paper.

\section{Background and Previous Work}

To efficiently address complex XML queries, it is important to: (i) quickly determine the structural relationship among any pair of tree nodes, (ii) efficiently find all occurrences of a structural relationship. We first provide background information on the importance of numbering schemes in determining structural relationships. We then discuss previous work on structural joins and indexing for XML documents.

Numbering Schemes. To locate all figures under a given section, the obvious but inefficient way is to traverse the whole subtree of the section (i.e., a top-down approach). Structural relationships can determined faster if a numbering scheme is embedded on the document's tree. One approach is to assign to each tree node three numbers, namely: the node's preorder and postorder ranks as well as its level in the XML tree $[13,40,24,1,19]$. In a preorder (postorder) traversal, a tree node is visited and assigned its preorder (postorder) rank before (after) its children are recursively traversed from left to right. The preorder traversal of a document's tree representation is equivalent to its textual order. If the document's textual representation is read sequentially, elements will be accessed in their preorder rank. Conversely, the document text can be recreated following a preorder traversal on its tree representation.

A node $v$ is an ancestor of a node $u$ iff $\operatorname{preorder}(v)<$ preorder $(u)$ and postorder $(v)>\operatorname{postorder}(u)$. If the (preorder, postorder) ranks are seen as an interval (clearly: $\operatorname{preorder}(v)<$ postorder $(v)$ ), then the ancestor contains the interval of the descendant. In the textual representation, the opening tag $\langle v\rangle$ is before $\langle u\rangle$ while the closing tag $\langle/ v\rangle$ is after $\langle/ u\rangle$. Furthermore, node $v$ is a parent of node $u$ if in addition level $(v)+1=\operatorname{level}(u)$.

While easily computed, the above numbering scheme is affected by document updates; node ranks change when inserting or deleting a node. A durable numbering scheme was recently proposed in [24]. In this scheme, each node is assigned a (start, end) interval (where end > start) such that: (i) for any node $u$ and its parent node $v$, the interval $(\operatorname{start}(u), \operatorname{end}(u))$ is contained in interval $(\operatorname{start}(v), \operatorname{end}(v))$; (ii) for two sibling nodes $u, v$, if $u$ is the predecessor of $v$ in preorder traversal, then $\operatorname{end}(u)<\operatorname{start}(v)$. The start numbers follow the pre-order traversal, but ranges of unused numbers are left between subsequent nodes to make room for future insertions. Node $v$ is an ancestor of a node $u$ iff $\operatorname{start}(v)<\operatorname{start}(u)<\operatorname{end}(v)$.

Figure 1 shows a sample XML document with its durable intervals (for simplicity, the tree level numbers are not shown). The root node is assigned interval $[1,2100]$, while its three children nodes have intervals $[10,600],[710,1200]$, and $[1400,2000]$. Ranges $[2,9],[601,709],[1201,1399]$, and [2001,2099] remain unused to handle future insertions. 


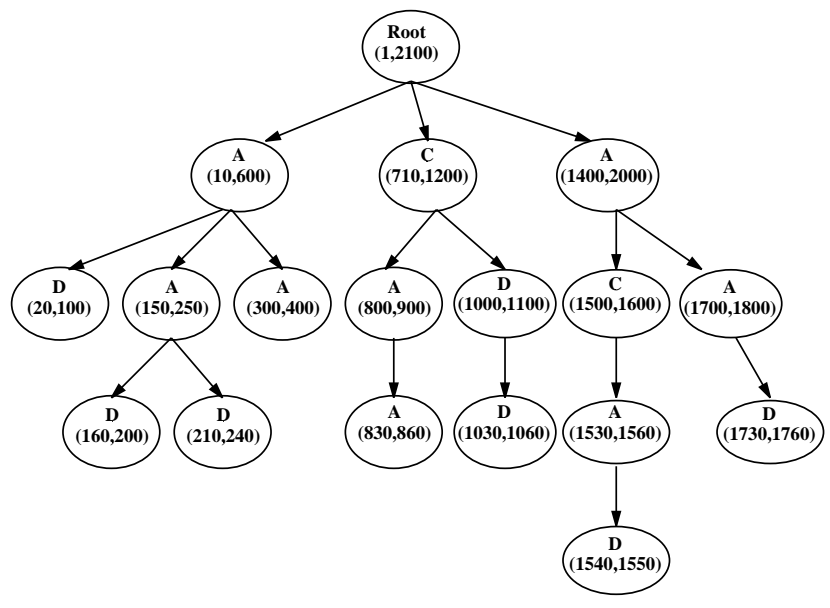

Figure 1: A sample XML document.

The proposed indexed join algorithms work with either numbering scheme. Note that under both (durable and non-durable) schemes, for any two distinct nodes $u, v$, the following hold: (i) the interval of $u$ is completely before or completely after the interval of $v$, or, (ii) the interval of $u$ contains or is contained by the interval of $v$. Hence intervals can never intersect partly.

Structural Joins. Structural join algorithms can take advantage of the numbering schemes previously discussed to expedite execution. Consider again our previous path query and assume that all sections with title Overview have been identified and stored in list $A$, while list $D$ contains all figures with caption Rtree. To answer the query, we must find all occurrences of section/figure pairs that satisfy the ancestordescendant relationship. This corresponds to computing a structural join [1] between the ancestor list $A$ and the descendant list $B$. (Structural joins are termed "containment queries" in [40] while [24] uses the terms EE-Join and EA-Join.) More formally, given two lists of elements $A$ and $D$, where each element is of the format: $\langle$ DocID, start, end, level $\rangle$ the (ancestor-descendant) structural join reports all pairs $\left(a_{i}, d_{j}\right)$ such that (i) $a_{i} . D o c I D=d_{j} . D o c I D$, and (ii) $a_{i}$. start $<d_{j}$. start and $a_{i}$. end $>d_{j}$.end. A parentchild structural join also requires that $a_{i}$.level $+1=$ $d_{j}$.level.

Structural joins are considered core operations in optimizing XML queries $[16,26,40,24,1]$. Various techniques have been proposed using traditional relational DBMS [16, 31,40] or native XML query engines [26]. For example, [40] proposes a variation of the merge join algorithm using multi-predicates. Nevertheless, this algorithm can perform a lot of unnecessary steps especially when computing parent-child relationships [1]. Similarly, the sort-merge join proposed in [24] may scan an element set many times during the join computation.
The Stack-Tree-Desc algorithm proposed by [1] represents the state-of-the-art in structural joins; it assumes that each element list is stored ordered on start and a stack mechanism is introduced to maintain elements that will be used later in the join. This leads to optimal join performance: only one sequential scan is performed on each list. Nevertheless, as it will be demonstrated in the next section, if an index is available on each ordered list, various elements that do not participate in the join will not be scanned. This can offer drastic improvement in the structural join performance.

More recent work in [5] extends the above algorithm to efficiently match more general selection patterns on elements that present specified tree structure relationships. Our solutions can be easily extended to incorporate the techniques in [5] as long as the element lists are appropriately indexed.

XML Indexing. Various techniques have been recently proposed for indexing XML data. We first discuss proposals that do not facilitate a numbering scheme $[18,17,22,27,28,12]$. These works create a structural summary of the XML document, in the form of a labelled directed graph, similar to the one used to model the XML document. The idea is to preserve all paths, while having fewer nodes and edges. Structural summaries extract structural information directly from the data. However, unlike a schema, they are not static, and thus may change with any update. They are approximate and they need to encode information about long, seldom-queried paths, leading to increased complexity. An index typically consists of a structural summary along with a stored mapping from summary nodes to data nodes. It can be used to evaluate path expressions directly (in a top-down approach) by pruning the search space. Nevertheless, since a structural summary does not contain all data nodes, many paths need to still be examined.

The solution proposed in [12] uses prefix-encoding. Paths are encoded as strings and inserted into a special index called Index Fabric which is based on Patricia tries. The index structure is tailored for path-queries that originate in the document root. Other paths require multiple index lookups or a post-processing phase. To remedy this drawback, the notion of refined path was proposed. However, the refined paths needs to be preselected before index loading time.

Durable node numbers provide an important advantage since they can serve as stable references for external indices $[24,11,19]$. In [24] $\mathrm{B}+$-trees are used to index the document elements and attributes; parentchild relationships are supported by the structure index. In [19] node intervals are represented as points in a higher dimensional space and an R-tree is used to store these points. In [11] we presented an indexing scheme based on multiversion indices (B+-trees and R-trees) and durable numbers to efficiently an- 
swer queries in multiversion documents. The scheme includes one index for the full document and a separate index for the elements of each tag (e.g. sections, chapters, figures). All indices are built on the element start numbers.

\section{$3 \quad$ Structural Joins using B+-trees}

Existing structural join algorithms [1,24] do not utilize index structures but sequentially scan the input lists. I/O's can be wasted for scanning elements that do not participate in the join. Recent durable numbering schemes have enabled indexing of XML files with mainstream indices, like $\mathrm{B}+$-trees and $\mathrm{R}$-trees. Maintaining such indices in the presence of document updates is trivial due to the reference permanence provided by the durable numbering. In this section we propose structural join algorithms using B+-trees; Rtree based structural joins are examined in section 4 .

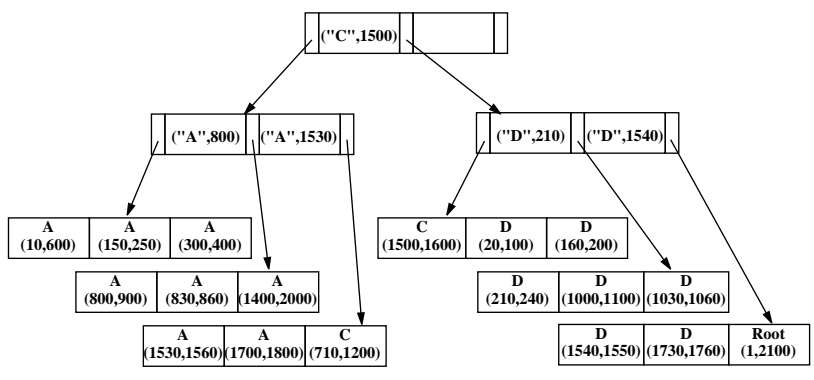

Figure 2: The B+-tree corresponding to the XML document of figure 1 .

For simplicity we consider a single, typically large, document. Extension to multi-document databases is trivial. Similarly, we concentrate on the ancestordescendant join; the parent-child join its a simple extension using the level numbers. (Hence in the rest, the DocID, level attributes are not shown but are implicitly assumed.) We assume that a separate index is used to cluster elements from the same tag (chapters, sections, figures, etc.) This index organization has been shown to be very efficient for simple path queries (i.e., when the subtree of an element does not contain elements from the same tag) [11]. In practice these multiple indices can be combined into a single index, simply by adding the tag name in the search key. An example appears in Figure 2; the index is built on the (tag, start) combination. (In a multidocument database, the search key would be: (DocID, tag, start)).

To motivate the use of indexing in structural joins, consider the examples illustrated in Figure 3. Thin line segments represent the (start,end) intervals of elements in the $A$ (ancestor) list, while thick line segments correspond to element intervals in the $D$ (descendant) list.

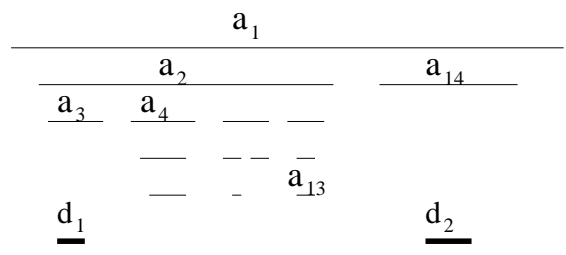

(a) Skip ancestor elements

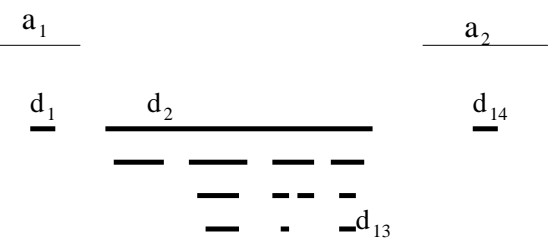

(b) Skip descendant elements

Figure 3: Motivation for using the $\mathrm{B}+$-tree index.

Figure 3a depicts a scenario where ancestor elements should be skipped. For this example, the sequential scan Stack-Tree-Desc algorithm from [1] will proceed as follows: (1) push $a_{1}, a_{2}$ and $a_{3}$ into the stack and join them with $d_{1} ;(2)$ pop $a_{3}$ and $a_{2}$ from the stack; (3) examine (push into and pop from the stack) elements $a_{4}$ through $a_{13}$ from the $A$ list; (4) push $a_{14}$ into the stack and then join $a_{14}$ and $a_{1}$ with $d_{2}$. Clearly, the third step is wasteful. We can utilize the $\mathrm{B}+$-tree index to omit the examination of elements $a_{4}$ through $a_{13}$ as follows: after $a_{2}$ is popped from the stack, directly go to $a_{14}$. Here $a_{14}$ is the $A$ element having the smallest start which is larger than $a_{2}$.end.

Figure $3 \mathrm{~b}$ illustrates the case where descendant elements should be avoided. Here, after $a_{1}$ is joined with $d_{1}$, the Stack-Tree-Desc algorithm will sequentially scan the descendant elements $d_{2}$ through $d_{13}$. Instead, the $\mathrm{B}+$-tree index can be used to jump directly to $d_{14}$, which is the element from list $D$ having the smallest start that is larger than $a_{2}$. start.

The complete $\mathrm{B}+$-tree based structural-join algorithm $\left(A n c_{-} D e s_{-} B+\right)$ follows. Since the leaf pages in each $\mathrm{B}+$-tree are linked together, we can view the leaf elements in that tree as a sorted list. Initially, variables $a$ and $d$ denote the first elements (having the smallest start) of the two sorted lists. Then the algorithm systematically moves $a$ and $d$ forward (to be the next element in their lists, etc.) and performs the join until one of the lists becomes empty. During the execution, a stack of elements from the $A$ list is maintained. That is, if all elements $a_{1}, a_{2}, \ldots, a_{k}$ where $a_{i}$ is before $a_{i+1}$, are ancestors of $d$, we push $a_{1}$ through $a_{k}$ into the stack before we join $d$ with them. This is similar to the Stack_Anc_Des algorithm processing. However, in algorithm $A n c_{-} D e s_{-} B+$, steps 11 and 15 utilize the $\mathrm{B}+$-trees to skip elements from the $A$ and $D$ lists, respectively. In practice, to avoid unnecessary B+-tree accesses, we first check whether the next ancestor element is in the same page $p$ as the previous ancestor 
element (by checking the last ancestor element in $p$ ).

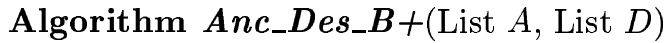

1. Let $a, d$ be the first elements of $A$ and $D$;

2. while ( not at the end of $A$ or $D$ ) do

3. if ( $a$ is an ancestor of $d$ ) then

4. Locate all elements in $A$ that are ancestors of $d$ and push them into stack;

5. Let $a$ be the last element pushed;

6. Output $d$ as a descendant of all elements in stack;

7. Let $d$ be the next element in $D$;

8. else if ( a.end $<$ d.start ) then

9. $\quad$ Pop all stack elements which are before $d$;

10. Let $l$ be the last element popped;

11. Let $a$ be the element in $A$ (locate using $\mathrm{B}+$ tree) having the smallest start that is larger than l.end;

12. else $/^{*} a$ is after $d$, or $a$ is a descendant of $d^{*} /$

13. Output $d$ as a descendant of all elements in stack;

14. if ( ancestor stack is empty) then

15.

Let $d$ be the element in $D$ (locate using $\mathrm{B}+$-tree) having the smallest start that is larger than a.start;

16. else

17. Let $d$ be the next element in $D$;

18. endif

19. endif

20. endwhile

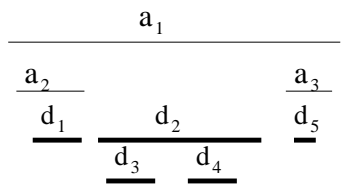

Figure 4: Skipping descendants when the stack is not empty leads to error.

Moreover, step 14 shows that in order for the algorithm to correctly skip elements in the descendant list $D$, the stack must be currently empty. Otherwise, we may skip erroneously some $D$ elements that need to be joined with the in-stack ancestor elements. Figure 4 shows an example of this situation. Here we assume the algorithm is currently checking ancestor $a_{3}$ against descendant $d_{2}$, while element $a_{1}$ is in stack. Since $a_{3}$ is after $d_{2}$, the algorithm goes to step 13 . Without step 14, we would then have continued to step 15 and skip descendants $d_{3}$ and $d_{4}$. However, this would fail to join $d_{3}$ and $d_{4}$ with $a_{1}$.

\subsection{Embedding the Containment Forest}

We present an easily maintainable enhancement that can further improve join performance. We first introduce the concept of the containment forest ( $C$-forest). A C-forest is a structure linking the elements from the same tag. Each element corresponds to a node in the structure and is linked to other elements from the same tag via parent, first-child and right-sibling pointers. In the rest, the terms parent, child and sibling refer to the C-forest structure.

Next, we discuss how these three pointers are determined for each node. Given nodes $n$ and $n_{p}$ from the same tag, node $n_{p}$ is called the parent of node $n$ iff: (a) $n_{p}$ is $n$ 's ancestor in the document tree (i.e. $n_{p}$. start $<$ n.start $<$ n.end $<n_{p}$. end $)$; and (b) there is no other ancestor node $n_{a}$ of $n$ from the same tag, such that $n_{p}$ is an ancestor of $n_{a}$. Symmetrically, we call $n$ a child of $n_{p}$. Given same-tag nodes $n$ and $n_{c}$, node $n_{c}$ is called the first-child of $n$ iff: (a) $n_{c}$ is a child of $n$; and (b) there does not exist another sametag node that is a child of $n$ which is before $n_{c}$ (node $n_{1}$ is before $n_{2}$ iff $n_{1}$.end $<n_{2}$.start). Finally, given same-tag nodes $n$ and $n_{s}, n_{s}$ is the right-sibling of $n$ iff: (a) $n$ and $n_{s}$ have the same parent; and (b) there is no same-tag node between them which has the same parent $\left(n_{2}\right.$ is between $n_{1}$ and $n_{3}$ iff $n_{1}$.end $<n_{2}$.start and $n_{2}$.end $<n_{3}$.start). Figure 5 depicts two C-forests, for the elements with tag $A$ and $D$ respectively, taken from the sample document in Figure 1.
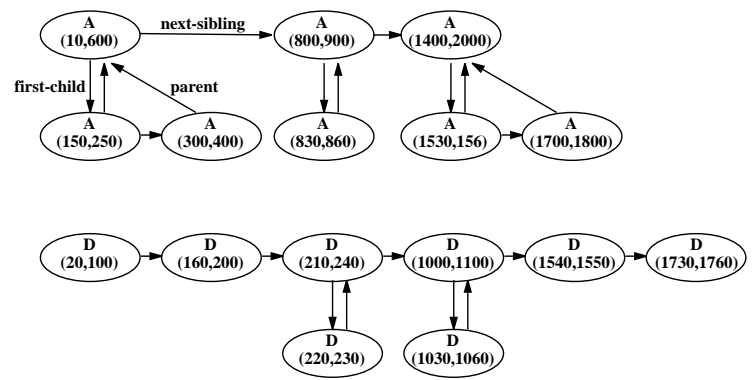

Figure 5: C-forests on tags $A$ and $D$ for the XML document of Figure 1.

A C-forest has the following properties:

- The (start, end) interval of each node contains all intervals in its subtree (hence the name containment forest).

- The start numbers in the forest follow a preorder traversal.

- The start (end) numbers of sibling nodes are in increasing order.

A C-forest for a given tag can be easily embedded within the B+-tree that indexes the tag's elements. This is accomplished by adding C-forest parent and 
next-sibling pointers among the leaf records of the $\mathrm{B}+-$ tree. First-child pointers are implicit because an element and its first child are always stored subsequently in the $\mathrm{B}+$-tree. Details are discussed in the next subsection.

The $A n c_{-} D e s_{-} B+$ join algorithm also applies when the $\mathrm{B}+$-tree is enhanced with the $\mathrm{C}$-forest pointers. One difference appears in step 11 that finds the element $a_{\text {new }}$ which has the smallest start larger than a.end. Consider the embedded $\mathrm{C}$-forest. The relationship between $a_{\text {new }}$ and $a$ is as follows: if $a$ has a right-sibling then $a_{\text {new }}=a$ asibling. If $a$ does not have a right-sibling, but $a$ 's parent has a sibling, then $a_{\text {new }}=a$ a.parent.sibling, and so on. If neither $a$ nor any of $a$ 's ancestors have a right-sibling, then the join algorithm completes since no other $A$ elements need to be examined.

Interestingly, at step 11, all of $a$ 's ancestors are in the running stack. Since each element has now a rightsibling pointer, the address of $a_{n e w}$ is identified directly, without any extra I/O. This improves algorithm Anc_Des_B+, since the B+-tree traversal is avoided in this case. Moreover, CPU time is gained as well. In the plain $\mathrm{B}+$-tree, even if all related pages are in memory, we still need to search (e.g. binary search) for the new ancestor element in these pages. This is avoided with the sibling pointers, since the readily available pointer to the new ancestor has both page ID and position within the page. In the rest we refer to the enhanced index and the improved join algorithm as $B+s p$-tree and $A n c_{-} D e s \_B+s p$, respectively.

\subsection{Dynamic Index Maintenance}

Maintaining the plain $\mathrm{B}+$-tree in the presence of document updates is straightforward: each element update translates to updating the element's durable start number. However, when the C-forest structure is embedded, the (C-forest) parent and right-sibling pointers need be maintained effectively at each element insertion/deletion. We first present the $\mathrm{B}+\mathrm{sp}$-tree insertion algorithm; deletion is examined afterwards.

attr)

Algorithm Insert_B $+\boldsymbol{s p}$ (int start, Attribute

1. Use the $\mathrm{B}+$-tree insertion algorithm to insert a new element (start,attr);

2. if ( leaf page overflow occurs ) then

3. for ( each element $i$ moved to a new page )

4. $\quad$ Adjust pointers pointing to $i$;

5. endfor

6. endif

7. Find the parent, left sibling, right sibling, and first child of the new element and adjust the corresponding C-forest pointers.

Basically, we first insert a new element into the $\mathrm{B}+$-tree using start as key, and then we adjust the right-sibling and parent pointers among leaf elements. The core step is step 7, which locates among the existing elements which will be the parent, left sibling, right sibling, and first child of the newly inserted element. Once the related elements are found, linking the new element into the C-forest is easy. (Identifying the possibly many children under the new element is performed starting from its first child and following right-sibling pointers. The right sibling pointer of the last child is set to NULL.)

We now focus on how to locate all related elements. To locate the left sibling of the new element $a$, we first use the $\mathrm{B}+$-tree to locate the element $e$ with the largest start which is smaller than a.start. Clearly, either $e$ is the parent of $a$, or $e$ is before $a$. If $e$ is $a$ 's parent, then $a$ does not have any left sibling. Otherwise, consider the parent of $e$. Again, either e.parent is a's parent, or it is before $a$. In the first case, $e$ is the left sibling of $a$. In the second case, we recursively consider the parent of e.parent, and so on. Eventually, if the highest ancestor of $e$ in the C-forest is before $a$, then this becomes the left sibling of $a$.

The procedure of finding the left sibling also identifies $a$ 's parent. To locate the right sibling of $a$, we use the $\mathrm{B}+$-tree to find the element $e$ with the smallest start that is greater than a.end. If e.parent is the same as the parent of $a, e$ is the right-sibling of $a$; otherwise $a$ does not have any right-sibling.

To locate the first child of $a$ is simpler. Since the leaf elements in the B+-tree are sorted by start number, we simply examine the element stored right after $a$. This element is either the first child of $a$, or after $a$. We can decide whether it is a first child simply by checking whether the end of the element is larger than a.end.

Finally, if a page overflow takes place after we insert the new element (steps 2-6), the B+-tree insertion algorithm will move some leaf records into another page. The pointers pointing to these moved records need to be adjusted, but this procedure is similar to the above.

The following algorithm discusses how to delete an element from the $\mathrm{B}+\mathrm{sp}$-tree:

\section{Algorithm Delete_B $+\boldsymbol{s p}$ ( Pointer $a)$}

1. Locate the left sibling and the first child of $a$;

2. Adjust the $\mathrm{C}$-forest pointers of the related elements;

3. Use the B+-tree deletion algorithm to delete element $a$;

4. if ( a leaf page underflow occurs ) then

5. for (each element $i$ moved to a new page)

6. $\quad$ Adjust pointers pointing to $i$;

7. endfor

8. endif

We first need to unlink the element $a$ from the Cforest. Again, the core step is to locate the related 
elements (parent, first child, left sibling, right sibling). Different from the insertion algorithm, there is no need to locate the parent and right sibling elements of $a$, since the pointers to these elements are already stored along with $a$. Once the related elements are identified, it is straightforward to adjust the C-forest pointers: for the children of $a$, we should set the parent pointers of them to point to a.parent; for the left sibling of $a$, we should set its right sibling pointer to point to the first child of $a$; for the last child of $a$, we should set its right sibling pointer to point to a.right. Next, we use the normal $\mathrm{B}+$-tree deletion algorithm to delete element $a$. If a page underflow occurs, records will be redistributed to a sibling page. In this case, we need to adjust the pointers which point to the elements that are redistributed.

To analyze the efficiency of the insertion/deletion algorithms of the $\mathrm{B}+\mathrm{sp}$-tree, we first define some terms. Given a C-forest, the depth of a node is the number of ancestors the node has. Then max-depth denotes the maximum depth of any node in the forest, while max-span corresponds to the maximum number of children under any node. These parameters depend on the document characteristics. Elements with longer intervals tend to attain many children elements and create deeper subtrees (and thus increase the maxspan and max-depth). The efficiency of the B+sp-tree insertion/deletion algorithm is given below. Due to space limitations the proof of the theorem is omitted.

Theorem 1 The amortized insertion/deletion cost of a $B+$ sp-tree is $O(h+s+d)$, where $h$ is the height of the $B+$-tree and $s, d$ are the max-span and max-depth of the embedded $C$-forest.

\section{Structural Join using R-trees}

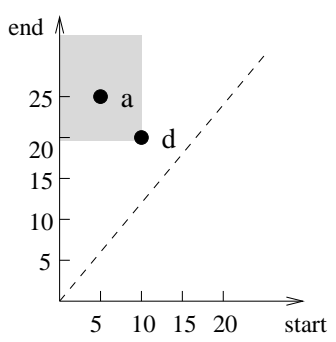

(a) two points

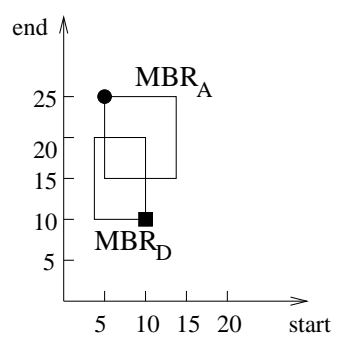

(b) two pages
Figure 6: The ancestor-descendant relationship in the transformed space.

Multidimensional indices (like the R-tree [20,6]) offer an alternative in indexing XML documents $[11,19]$. Consider the (start,end) interval of element $e$. This interval is mapped to a point (e.start, e.end) in the 2dimensional space which is then indexed by an R-tree. Figure 6a depicts two points corresponding to elements $a:(5,25)$ and $d:(10,20)$. Since end $>$ start for any element, all points in this representation lie above the diagonal. Moreover, the following properties hold:

- An element $a$ is an ancestor of element $d$ iff the corresponding point of $a$ is located on the upper left side of point $d$ (Figure 6a).

- Let $A, D$ be two R-tree pages that store points from the ancestor and descendant lists, respectively. Let $M B R_{A}$ and $M B R_{D}$ be the minimum bounding rectangles of these pages. Page $A$ may contain an ancestor of some point in page $D$ iff the upper left corner of $M B R_{A}$ (the black dot in figure $6 \mathrm{~b}$ ) is located on the upper left side of the lower right corner of $M B R_{D}$ (the black square in figure $6 \mathrm{~b})$.

Typically, spatial join algorithms join two sets of rectangular objects and report pairs of objects that intersect. If each set is indexed by an R-tree, a synchronized tree traversal $(S T T)$ is followed $[4,21]$. Initially, the two root pages are joined. To join a pair of index pages, every element in the first page is joined with every element in the second page, if they intersect. Eventually, at the leaf level, two objects are joined if they intersect. The R-tree based structural join algorithm that we propose also uses STT. The difference is that instead of using the intersection condition, the condition to join two pages $A$ and $D$ is that $A$ 's upper left corner is located to the upper left of $D$ 's lower right corner; and the condition to join two objects $a$ and $d$ is that $a$ is located to the upper left of $d$.

Similarly, we can use an R-tree to index the element (start,end) ranges as 1-dimensional intervals. To perform a structural join on two interval based R-trees, we utilize existing join algorithms with slight modification: two objects with intervals $r_{1}$ and $r_{2}$ are joined as long as $r_{1}$ contains $r_{2}$. In our experiments we implemented both R-tree approaches.

\section{Problem Variations}

\subsection{Parent-Child Join}

All the three ancestor-descendant structural join algorithms can be directly used to answer parent-child joins. An additional condition is performed on the levels of each (ancestor,descendant) pair found.

\subsection{Self Joins}

If both ancestor and descendant elements have the same tag (self join), a simpler algorithm applies. For example, in the self join case, if we maintain two pointers $a$ and $d$ as the previous algorithms do, the two pointers may point to the same element, a case which (for simplicity) is omitted from the previous algorithms. Moreover, the B+-tree index algorithms were used to skip sub-trees when possible. In the selfjoin case, however, as long as a sub-tree has more than 
one element, every element of the sub-tree will appear in the join result (e.g. every element is a descendant of the sub-tree's root). We could use the R-tree join algorithm. However, there is no guarantee that each disk page will be examined at most once.

We hereby propose an non-indexed self join algorithm, assuming that the elements are sorted in ascending order of start:

\section{Algorithm Self_Anc_Des( List $A)$}

1. Let $a$ be the first element in $A$;

2. while ( not at the end of $A$ )

3. Pop all stack elements which are before $a$;

4. Output $a$ as a descendant of the remaining elements in stack;

5. Push $a$ into stack;

6. Let $a$ be the next element in $A$.

7. endwhile

The algorithm traverses the list exactly once, while maintaining a stack in which any adjacent pair of elements has parent-child relationship. For each element $a$ being examined, we first pop from the stack those elements that are before $a$ since they will not join with any later elements. We then join $a$ with the in-stack elements and finally push $a$ into the stack.

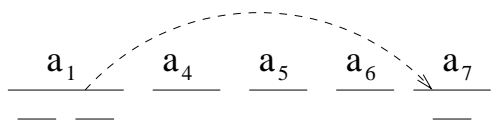

Figure 7: The next-useful-tree pointer helps to speedup self join.

Discussion: If there are many elements which do not have any ancestor or descendant at all (e.g. elements $a_{4}, a_{5}, a_{6}$ in Figure 7), algorithm Self_Anc_Des is not efficient since it has to go through the whole list. One possible optimization is to link useful trees (i.e. trees having at least two elements): in figure 7 a link connects $a_{1}$ to $a_{7}$. Algorithm Self_Anc_Des will then skip elements which do not participate in the join.

\subsection{Structural Join in a Pipelining Environ- ment}

We consider the structural join problem in a different scenario: the input lists are not known beforehand, but are generated by previous-stage execution modules in a pipelined environment. For example, in figure 8, the inputs to the join arrive from producer modules 1 and 2. In this scenario, the structural join module can not benefit from pre-built indices. Thus, if the structural join module cannot send any feedback to the producer modules, the best approach is to use the non-indexed sequential algorithm proposed in [1].

However, if feedbacks are allowed (i.e., the join module is "active"), we can still use the Anc_Des_B+

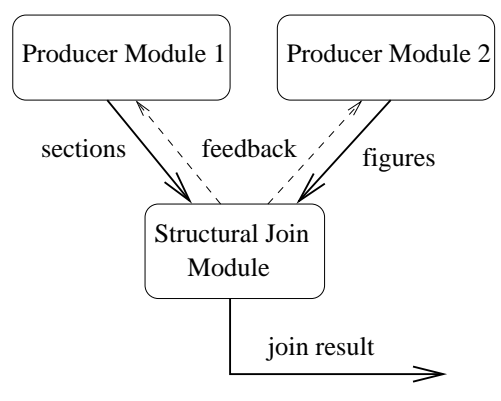

Figure 8: Structural join engine.

algorithm to improve the performance, even though the $\mathrm{B}+$-tree indices are not available to the structural join module. The idea is as follows. In steps 11 of algorithm Anc_Des_B+, we skip ancestors by locating (using the $\mathrm{B}+$-tree) the element which the smallest start larger than a.end, where $a$ is the ancestor being examined currently. In the pipelining environment, the join module should send a feedback asking producer module 1 to skip ancestors accordingly. Similarly, in step 15 , the join module should ask producer module 2 to skip descendants. Simply not sending the skipped data across execution modules would reduce the size of intermediate results. Moreover, a clever producer module might be able to use this feedback to further optimize its data generation plan.

\section{Performance Analysis}

We compare the performance of the non-indexed structural join algorithm (Stack-Tree-Desc [1]), the B+-tree join algorithm (Anc_Des_B+), the B+-tree join with sibling pointers (Anc_Des_B+sp), as well as two Rtree based algorithms (using 1-dimensional intervals and 2-dimensional points respectively). We used the $\mathrm{R}^{*}$-tree [6] as the R-tree implementation. For each R-tree approach, we checked both breadth-first join and depth-first join. The results were very close so for simplicity we report only the depth-first joins. Moreover, as will be explained later in this section, we also implemented an optimization of Anc_Des_B+sp, using a partial list (i.e., the most important) of the sibling pointers. The notations used in the performance figures are described in table 1.

\subsection{Experimental Setup}

For the purposes of our experiments we have implemented a storage manager with an LRU replacement policy as well as the B+ tree index structure, and its enhancement using the C-forest. Both access methods provide bulk-loading facilities. All the algorithms were implemented in $\mathrm{C}++$ and compiled using the GNU $\mathrm{C}++$ compiler, under Linux Mandrake 8.0. The experiments were contacted on a Pentium III $1.2 \mathrm{GHz}$ processor with $1 \mathrm{G}$ of main memory.

We used synthetic data for all our experiments in 


\begin{tabular}{|c|c|c|}
\hline Notation: & Meaning: & Section: \\
\hline$n o \_i n d e x$ & structural join using sequential scan (Stack-Tree-Desc [1]) & 2 \\
\hline$B+$ & structural join using B+ tree indices (Anc_Des_B+) & 3 \\
\hline$B+s p$ & structural join using B+ trees with sibling pointers (Anc_Des_B+sp) & 3.1 \\
\hline$B+p s p$ & structural join using B+ trees and partial list of sibling pointers & 6.2 \\
\hline$R^{*}$ & structural join using $\mathrm{R}^{*}$-trees with 1-dimensional intervals & 4 \\
\hline$R^{* 2}$ & structural join using $\mathrm{R}^{*}$-trees with 2-dimensional points & 4 \\
\hline
\end{tabular}

Table 1: Implemented Algorithms.

<!ELEMENT department (name, email?, employee+)>

$<$ !ATTLIST department nodeID ID \#REQUIRED startPos CDATA \#REQUIRED endPos CDATA \#REQUIRED level CDATA \#REQUIRED dOCID CDATA \#FIXED "1">

<!ELEMENT employee (employee*, name+, email?)>

$<$ !ATTLIST employee nodeID ID \#REQUIRED startPos CDATA

\#REQUIRED endPos CDATA \#REQUIRED level CDATA \#REQUIRED

dOCID CDATA \#FIXED "1">

$<$ !ELEMENT name (\#PCDATA)>

$<$ !ATTLIST name nodeID ID \#REQUIRED startPos CDATA

\#REQUIRED endPos CDATA \#REQUIRED level CDATA \#REQUIRED

dOCID CDATA \#FIXED "1">

$<$ !ELEMENT email (\#PCDATA)>

$<$ !ATTLIST email nodeID ID \#REQUIRED startPos CDATA

\#REQUIRED endPos CDATA \#REQUIRED level CDATA \#REQUIRED dOCID CDATA \#FIXED "1">

Figure 9: The DTD for the experimental data.

order to control the structural and consequently join characteristics of the XML documents. We generated several XML files using the IBM XML data generator [37] providing it with the DTD presented in figure 9. Most of the XML trees that we generated had depth seven. The derived documents were further processed to obtain the desired data workloads.

We parsed the generated XML files in order to add the (start,end) numbers to each element node using an event-based XML parser [36] that we implemented in Java. The parser uses a stack that grows to the maximum nesting-depth of the XML nodes and assigns the durable numbers in two passes over the XML file. One pass allocates the durable numbers while the second pass integrates them into the XML text file as attributes to each node. Subsequently, we parsed the generated file to create the XML node lists. The elements of each list include the attributes of the associated node, as well as its (start,end) interval. All lists are stored in binary format. The data are then bulkloaded into the $\mathrm{B}+$ trees. For the experiments we used a page size of $8 \mathrm{~K}$. The size of the lists varies from $20 \mathrm{M}$ to $100 \mathrm{M}$. To measure the join performance we count the CPU time of each algorithm as well as the number of I/O's. Note that because of the merge join characteristics of the structural join, most I/O's are non-sequential. We actually tested all algorithms for their I/O characteristics and found that between $75 \%$ and $80 \%$ of their I/O activity was random. Each group of experiments was performed using different data sets (i.e. XML documents), so as to have a finer control on the elements that were joined subsequently.

\subsection{Join Performance Comparison}

Determining the Buffer Pool Size: We started the experiments by varying the buffer pool size. Precisely, we tried sizes of $20,40,80,100,150$ and 200 pages. As far as the structural no-index join algorithm is concerned, performance is not greatly affected by the buffer size. We observed the same behavior for the $\mathrm{B}$ +-tree algorithms, when the buffer pool contains 80 or more pages. The reasons are:

1. The noindex join algorithm scans the lists only once. As a result, after a page is visited for the first time and then flushed out, it will not be requested again in the future.

2. The same holds for the B+-tree algorithms. The difference in performance for smaller sized buffer pools is attributed to the fact that some index pages might be flushed out and then requested again. However, with larger buffer pool sizes and with LRU policy, chances are that the index pages will remain in the buffer pool.

Hence in the following experiments the buffer pool size is fixed to 80 pages.

\section{Join Performance}

We performed experiments varying the relative join characteristics of the ancestor and descendant lists. We also varied the result sizes. To identify the performance characteristics of each algorithm, we first examined their behavior by varying the percentage of elements from the ancestor list that join with descendants. In this scenario, skipping (mainly) ancestors becomes gradually more important. We then considered the alternative case of varying the percentage of descendant elements that participate in the join result. Here, the ability to (mainly) skip descendants will improve join performance. Finally, we examined scenarios where skipping in both lists is possible.

Skipping Ancestors: In the first group of experiments we kept the percentage of the descendants that are joined with at least one ancestor high (90\%), and we varied the percentage of the joined ancestors. For that purpose, we started with two lists where both ancestors and descendants are joined equally high (90\%) and gradually reduced the percentage of the joined ancestors. This reduction is performed by effectively removing elements from the descendant file (while at the 
same time maintaining the descendant join percentage around $90 \%$ ). The join performance is shown in table 2.

As can be seen from the table, all the algorithms, except the R-tree based ones, perform similarly. The performance loss that the $\mathrm{B}+\mathrm{sp}$ algorithm faces in comparison with the $\mathrm{B}+$-tree and the noindex joins is attributed to the increase of the file size due to the addition of sibling pointers. For the B+-tree, the only overhead that occurs is the initial loading of the index pages. However, this is quite small. The R-tree algorithms very often need to examine a page multiple times since their data pages are not completely clustered by the element startnumbers.

$B+p s p$ Optimization: We thus offer an optimization that can combine the advantages of the plain $\mathrm{B}+-$ tree as well as the sibling pointers. Typically, the sibling pointer for element $e$, is beneficial when it points to an element that is in a different page than $e$. Hence we can reduce the additional space overhead by maintaining only the useful sibling pointers. Experimental measurements that we contacted on our data, showed that on average a very small number of sibling pointers (usually $1 \%$ to $2 \%$ of the total number of sibling pointers) actually points to an element on a different page. We experimented with two different policies on how to decide which sibling pointers to maintain. One policy keeps the sibling pointer of an element whose subtree contains more than a fixed number, say 100, elements in the C-forest. The other policy kept the pointers that lead to a different page. The latter was more efficient and was implemented in the rest of our experiments $(\mathrm{B}+\mathrm{psp})$. In the results depicted in table 2 the $\mathrm{B}+$ psp algorithm performs equally well as the $\mathrm{B}+$-tree join. However, as it will be shown later it can get even better performance. In the following experiments we discuss the optimized version of the sibling-pointer join (B+psp).

Skipping Descendants: Next, we kept the percentage of ancestors that are joined with at least one descendant high (85\%), and varied the percentage of the joined descendants. In this case, it becomes beneficial to be able to skip descendant nodes. The join performance is shown in figure 10 .

As the percentage of joined descendants decreases the $\mathrm{B}+$ and $\mathrm{B}+\mathrm{psp}$ algorithms outperform the no_index join. This is because the latter is constrained to sequentially scan the two lists. The B+-tree algorithms avoid descendants by skipping elements. The R-tree algorithms performed much worse than the no_index join (around $80 \%$ more time) and are not depicted. The largest proportion (99\%) of the join time is consumed in page IOs. More, the noindex consumes more $\mathrm{CPU}$ time than the $\mathrm{B}+$ join which in turn is more $\mathrm{CPU}$ consuming than the $\mathrm{B}+\mathrm{psp}$ join.

Actually, the gain in performance when using the indexed joins is greater in the case of excluding descen-

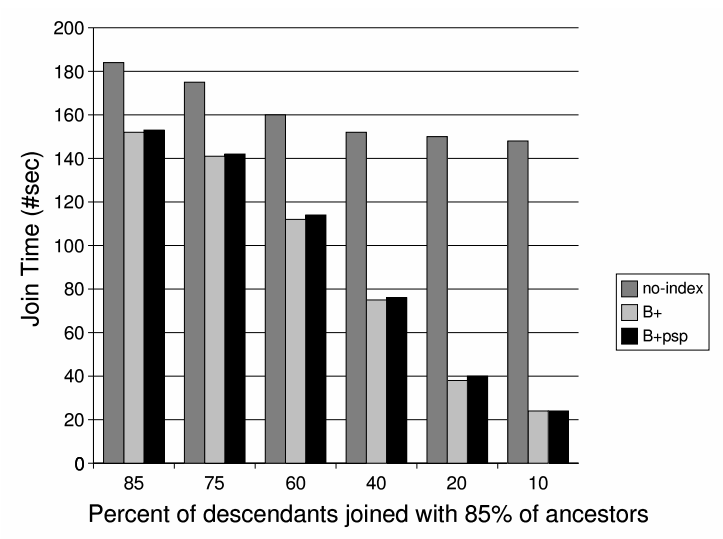

Figure 10: Effect of skipping only descendants.

dants than in the case of excluding ancestors. This is attributed to the fact that, when in need to exclude ancestors, the algorithms cannot take full advantage of the place of the current descendant to decide where the next candidate ancestor resides. Instead they can only jump past the current ancestor node. That may lead to visiting ancestor nodes that turn out not to join with any descendant nodes.

Skipping both Ancestors and Descendants: In the next group of experiments we varied the percentage of both ancestors and descendants that can be joined. Among all experiments we only show the results where $1 \%$ of ancestors elements participate in the join, while varying the number of descendants. The results are shown in figure 11 . Clearly, the B+-tree and $\mathrm{B}+$ psp perform better than the no_index and R-tree joins. Actually, here, the R-tree joins are similar to the noindex case, since only few ancestors join and the R-trees can locate them quickly. When the number of descendants decreases, the B+psp tree performs better than the $\mathrm{B}+$ tree. This is because the algorithm takes advantage of the sibling pointers at the ancestor nodes when it needs to access pages where the plain $\mathrm{B}+$ tree would need to traverse full index paths to find them.

Comparison of $\mathrm{B}+$-tree and $\mathrm{B}+$ psp algorithms: In the above experiments the performance of the $\mathrm{B}+$-tree and $\mathrm{B}+\mathrm{psp}$ algorithms were very close. This is not surprising given that the two algorithms utilize similar techniques and data structures to skip nodes. However, there is a difference which, for certain XML documents, might prove substantial. When in need to skip ancestor nodes, the $B+$ tree algorithm relies on the existent index structure, while the $B+p s p$ algorithm utilizes the pointer sibling node. If the join selectivity is very small the first algorithm will access a lot of index pages (leaf ones for the most part). This behavior is shown in figure 12 which shows substantial 


\begin{tabular}{|c|c|c|c|c|c|c|}
\hline Join Ancestors & no_index & $\mathrm{B}+$ & $\mathrm{B}+\mathrm{psp}$ & $\mathrm{B}+\mathrm{sp}$ & $\mathrm{R}^{*}$ & $\mathrm{R}^{*} 2$ \\
\hline $90 \%$ & 182 & 180 & 180 & 190 & 230 & 228 \\
\hline $70 \%$ & 150 & 149 & 150 & 155 & 198 & 196 \\
\hline $55 \%$ & 132 & 130 & 130 & 140 & 176 & 178 \\
\hline $40 \%$ & 109 & 108 & 108 & 114 & 160 & 156 \\
\hline $25 \%$ & 86 & 84 & 84 & 90 & 132 & 130 \\
\hline $15 \%$ & 74 & 67 & 67 & 70 & 122 & 119 \\
\hline
\end{tabular}

Table 2: Effect of skipping only ancestors in join performance.

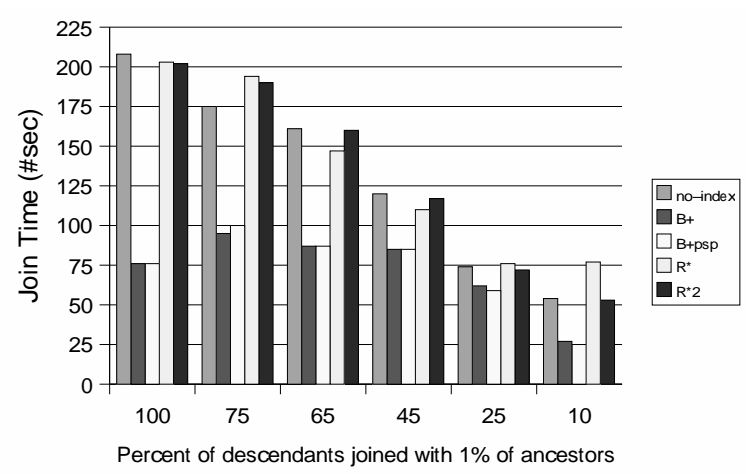

Figure 11: Effect of skipping both ancestors and descendants.

improvement in join performance (up to $18 \%$ ) of the $\mathrm{B}+$ psp over the $\mathrm{B}+$-tree approach. In this particular experiment, the algorithms had to skip many ancestor nodes. The $\mathrm{B}+$ tree algorithm had to access directory nodes before finding the data page, while the $\mathrm{B}+\mathrm{psp}$ algorithm was using the existing sibling pointers to go directly to the data pages that had joining nodes.

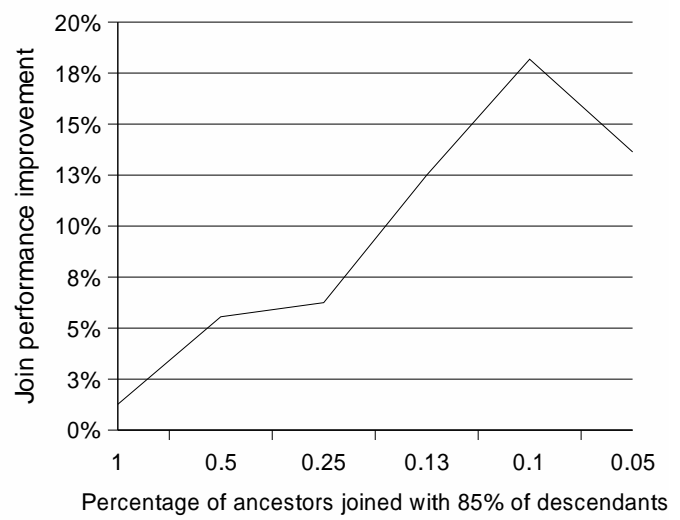

Figure 12: Comparing $\mathrm{B}+$ and $\mathrm{B}+\mathrm{psp}$.

\section{Conclusions \& Future Work}

In this paper, we proposed various indexed structural join algorithms over XML data. Performance results prove that the indexed algorithms are more robust than the state-of-art algorithms, which do not utilize index structures. Among the indexed approaches, the $\mathrm{B}+$-tree with sibling pointer performs the best.
We have also shown that traditional indexing schemes are easily enhanced to support our structural join algorithm-besides the more conventional valuebased searches. Finally, we have shown how these indexing schemes can be made durable, and thus support updates on XML documents, or the preservation of multiple versions of these documents [11]. These results are applicable to both the situation where native storage managers are used to store and query XML documents, and the situation where more traditional database systems are used for that purpose.

As future work to this paper, we plan to examine the indexed structural join over multi-versioned documents.

\section{References}

[1] S. Al-Khalifa, H. V. Jagadish, N. Koudas, J. M. Patel, D. Srivastava and Y. Wu, "Structural Joins: A Primitive for Efficient XML Query Pattern Matching", Proc. of ICDE, 2002.

[2] S. Abiteboul, D. Quass, J. McHugh, J. Widom and J. L. Wiener, "The Lorel Query Language for Semistructured Data", Journal on Digital Libraries 1(1), 1997.

[3] B. Becker, S. Gschwind, T. Ohler, B. Seeger and P. Widmayer, "An Asymptotically Optimal Multiversion B-Tree", VLDB Journal 5(4), 1996.

[4] T. Brinkhoff, H. Kriegel and B. Seeger, "Efficient Processing of Spatial Joins using R-trees", Proc. of SIGMOD, 1993.

[5] N. Bruno, N. Koudas, and D. Srivastava, "Holistic Twig Joins: Optimal XML Pattern Matching", Proc. of SIGMOD, 2002.

[6] N. Beckmann, H. P. Kriegel, R. Schneider and B. Seeger, "The R*-tree: An Efficient and Robust Access Method for Points and Rectangles", Proc. of SIGMOD, 1990.

[7] S. Ceri, S. Comai, E. Damiani, P. Fraternali, S. Paraboschi and L. Tanca, "XML-GL: A Graphical Language for Querying and Restructuring XML", Proc. of $W W W$ Conf., 1999.

[8] D. Chamberlin, J. Robie, D. Florescu, "Quilt: An XML Query Language for Heterogeneous Data Sources", Proc. of WebDB, 2000.

[9] S. Chawathe, A. Rajaraman, H. Garcia-Molina and J. Widom, "Change Detection in Hierarchi- 
cally Structured Information", Proc. of SIGMOD, 1996.

[10] S.-Y. Chien, V.J. Tsotras and C. Zaniolo, "Efficient Management of Multiversion Documents by Object Referencing", Proc. of VLDB, 2001.

[11] S.-Y. Chien, V.J. Tsotras, C. Zaniolo, and D. Zhang, "Efficient Complex Query Support for Multiversion XML Documents", Proc. of EDBT, 2002.

[12] B.F.Cooper, N. Sample, M.J.Franklin, G.R.Hjatlason, and M. Shadmon, "A fast index for semistructured data", Proc. of $V L D B$, 2001.

[13] P.F. Dietz and D. Sleator, "Two Algorithms for Maintaining Order in a List", Proc. of STOC, 1987.

[14] A. Deutsch, M. Fernandez, D. Florescu, A. Levy and D. Suciu, "A Query Language for XML", Proc. of WWW Conf., 1999.

[15] M. Fernandez and D. Suciu, "Optimizing Regular Path Expressions Using Graph Schemas", Proc. of ICDE, 1998.

[16] D. Florescu and D. Kossman, "Storing and Querying XML Data Using an RDBMS", IEEE Data Engineering Bulletin 22(3), 1999.

[17] A. Gionis, M. Garofalakis, R. Rastogi, S. Seshadri and K. Shim, "XTRACT: A system for extracting document type descriptors from XML documents", Proc. of SIGMOD, 2000.

[18] R. Goldman and J. Widom, "Dataguides: Enabling query formulation and optimization in semistructural databases", Proc. of VLDB, 1997.

[19] T. Grust, "Accelerating XPath Location Steps", Proc. of SIGMOD, 2002.

[20] A. Guttman, "R-trees: A Dynamic Index Structure for Spatial Searching", Proc. of SIGMOD, 1984.

[21] Y. Huang, N. Jing and E. Rundensteiner, "Spatial Joins Using R-trees: Breadth-First Traversal with Global Optimizations", Proc. of VLDB, 1997.

[22] R. Kaushik, P. Shenoy, P. Bohannon and E. Gudes. "Exploiting Local Similarity for Indexing Paths in Graph-Structured Data", Proc. of ICDE, 2002.

[23] A. Kumar, V. J. Tsotras and C. Faloutsos, "Designing Access Methods for bitemporal Databases", IEEE TKDE 10(1), 1998.

[24] Q. Li and B. Moon, "Indexing and Querying XML Data for Regular Path Expressions", Proc. of $V L D B, 2001$.
[25] D. Lomet and B. Salzberg, "Access Methods for Multiversion Data", Proc. of SIGMOD, 1989.

[26] J. McHugh and J. Widom, "Query optimization for XML", Proc. of VLDB, 1999.

[27] T. Milo and D. Suciu, "Index structures for path expressions", Proc. of ICDT, 1999.

[28] S. Nestorov, J. Ullman, J. Weiner and J. Chawathe. "Representative Objects: Concise representations of semistructured hierarchical data", Proc. of ICDE, 1997.

[29] B. Salzberg and V. J. Tsotras, "Comparison of Access Methods for Time-Evolving Data", ACM Computing Surveys 31(2), 1999.

[30] J. Shanmugasundaram, K. Tufte, G. He, C. Zhang, D. J. DeWitt and J. F. Naughton, "Relational Databases for Querying XML Documents: Limitations and Opportunities", Proc. of $V L D B$, 1999.

[31] J. Shanmugasundaram, K. Tufte, C. Zhang, G. He, D.J. DeWitt and J.F. Naughton, "Relational Databases for Querying XML Documents: Limitations and Opportunities", Proc. of VLDB, 1999.

[32] F. Tian, D. J. DeWitt, J. Chen and C. Zhang, "The Design and Performance Evaluation of Various XML Storage Strategies", http://www.cs.wisc.edu/niagara/Publications .html

[33] V.J. Tsotras and N. Kangelaris, "The Snapshot Index: An I/O-Optimal Access Method for Timeslice Queries", Information Systems 20(3), 1995.

[34] Y. Tao and D. Papadias, "MV3R-Tree: A SpatioTemporal Access Method for Timestamp and Interval Queries", Proc. of VLDB, 2001.

[35] P. Varman and R. Verma, "An Efficient Multiversion Access Structure", IEEE TKDE 9(3), 1997.

[36] SAX (Simple API for XML), http://sax. sourceforge.net

[37] IBM XML Generator, http://www.alphaworks. ibm.com/tech/xmlgenerator

[38] World Wide Web Consortium, "XML Path Language (XPath)", version 1.0, Nov 16, 1999. http:// www.w3.org/TR/xpath.html

[39] World Wide Web Consortium, "XQuery 1.0: An XML Query Language", W3C Working Draft Jun 7, 2001 (work in progress). http://www.w3.org/TR/xquery/

[40] C. Zhang, J.F. Naughton, D.J. DeWitt, Q. Luo and G.M. Lohman, "On Supporting Containment Queries in Relational Database Management Systems", Proc. of SIGMOD, 2001. 